\title{
Association between Variation of Troponin and Prognosis of Acute Myocardial Infarction before and after Primary Percutaneous Coronary Intervention
}

\author{
Xiaoxiao Zhao, ${ }^{1}$ Ying Wang, ${ }^{1}$ Chen Liu, ${ }^{1}$ Peng Zhou, ${ }^{1}$ Zhaoxue Sheng, ${ }^{1}$ Jiannan Li, ${ }^{1}$ \\ Jinying Zhou, ${ }^{1}$ Runzhen Chen $\mathbb{D}^{1},{ }^{1}$ Yi Chen, ${ }^{1}$ Hanjun Zhao, ${ }^{1,2}$ and Hongbing Yan $\mathbb{D}^{1,2}$ \\ ${ }^{1}$ Department of Cardiology, Fuwai Hospital, National Center for Cardiovascular Diseases, Peking Union Medical College, \\ Chinese Academy of Medical Sciences, Beijing, China \\ ${ }^{2}$ Fuwai Hospital Chinese Academy of Medical Sciences, Shenzhen, China
}

Correspondence should be addressed to Hongbing Yan; hbyanfuwai2018@163.com

Received 28 April 2020; Accepted 30 June 2020; Published 26 July 2020

Academic Editor: Salvatore De Rosa

Copyright ( 2020 Xiaoxiao Zhao et al. This is an open access article distributed under the Creative Commons Attribution License, which permits unrestricted use, distribution, and reproduction in any medium, provided the original work is properly cited.

Background. Circulating levels of cardiac troponin I (cTnI) after ST-segment elevation myocardial infarction (STEMI) were considered as prognostic factors for predicting the incidence of major adverse cardiovascular events (MACE). $\triangle \mathrm{cTnI}$ is the difference between peak cTnI after primary percutaneous coronary intervention (PPCI) and cTnI on initial admission. Purpose. This study aimed to assess the relationship between $\triangle \mathrm{cTnI}$, the ratio of $\triangle \mathrm{cTnI}$ to $\mathrm{cTnI}$ on initial admission, and the incidence of MACE during the follow-up period. Methods. A total of 2596 patients with cTnI measured upon admission and one-time measurement of $\mathrm{cTnI}$ during hospitalization were enrolled. Results. In the adjusted models of the survival receiver operating characteristic (ROC) curve, $\triangle \mathrm{cTnI}$ and the ratio of $\triangle \mathrm{cTnI}$ to $\mathrm{cTnI}$ on initial admission have stronger discrimination power of MACE (area under curve (AUC) 0.730 and 0.717) compared with peak cTnI after PPCI and cTnI at admission (AUC 0.590, 0.546). Multivariate Cox regression analysis identified $\triangle \mathrm{cTnI}$ (hazard ratio (HR) 1.018, 95\% confidence interval (CI) 1.001 to 1.035 ) as a relevant factor for MACE during follow-up. $\triangle \mathrm{cTnI}$ was divided into quartiles, and maximum $\triangle \mathrm{cTnI}$ between 4.845 and $19.073 \mathrm{ng} / \mathrm{ml}$ comprised more patients with anterior wall myocardial infarction $(p<0.001)$, higher GRACE score $(p=0.038)$, CKMB $(p=0.023)$, and Myoglobin $(p<0.001)$. On the K-M survival curves, the incidence of MACE, mortality, and angina pectoris were significantly higher in the group with maximum $\triangle \mathrm{cTnI}(p=0.035,0.049,0.026)$. Conclusion. The $\triangle \mathrm{cTnI}$ level and the ratio of $\triangle c T n I$ have stronger discrimination power of predicting the incidence of MACE. The group with maximum $\triangle c T n I$ has higher incidence of MACE, mortality, and angina pectoris during the follow-up period.

\section{Introduction}

Cardiac troponin I (cTnI) is a highly specific and sensitive biomarker of cardiac injury and a regulatory protein with cytosolic and structural compartments within the cardiac myocytes [1]. cTnI is a heart-specific protein released in the circulation upon myocardial injury and plays a significant role in the regulation of muscle contraction and cardiac troponins [2]. Conventional assay measurements of cTnI levels are routinely used to rule out acute myocardial infarction (AMI) and to assess the 30-day and 90-day prognoses of patients presenting with acute coronary syndrome
(ACS) $[3,4]$. Various studies [5-7] have reported that the circulating levels of cTnI after ST-segment elevation myocardial infarction (STEMI) are related to clinical outcomes and considered a prognostic predictor of major adverse cardiovascular events (MACE). However, the relationship of the cTnI level difference between the pre- and post-primary percutaneous coronary intervention (PPCI) is not well defined. To address this knowledge gap, this study aimed to explore the prognostic value of the cTnI level difference between PPCI peak cTnI and first admission cTnI to MACE during follow-up in a contemporary, homogeneous, and well-defined cohort of patients with STEMI undergoing PPCI. We investigated the 
correlation of first cTnI, peak cTnI after PPCI, $\triangle \mathrm{cTnI}$, and the ratio of $\triangle c T n I$ to $c T n I$ on initial admission, considering coronary angiography and echocardiography data and incidence of MACE. Moreover, we compared the discriminatory value of all four parameters in discriminating MACE.

\section{Methods}

2.1. Subjects. From a total of 4064 patients who presented at Fuwai Hospital in Beijing, China, between January 2010 and July 2018, 3586 consecutive STEMI patients (2713 men; age: 24-97 years) were enrolled (478 patients who were lost to follow-up were excluded from the study). All patients were referred to the coronary catheterization center with the diagnosis of acute STEMI fulfilling the criteria for PPCI according to the guidelines $[8,9]$. The study was approved by the Ethics Committee of Fuwai Hospital, and all patients gave informed consent for coronary angiography and PPCI.

Patient records including demographics, medical history, physical examination, blood test results, electrocardiography (ECG), echocardiography data, and discharge medication regimen were reviewed. Blood testing was performed at the clinical laboratory in Fuwai Hospital. Blood samples for cTnI measurement were acquired on admission and after PPCI. Patients were required to have at least one measurement of the cTnI level during hospitalization. However, of the 3586 patients, those who did not have a valid cTnI result and those with missing postdischarge follow-up data were also excluded. Finally, 2598 patients were included in the analysis. The study flow chart is shown in Figure 1.

2.2. Definitions. In this study, $\triangle c T n I$ was defined as the value calculated as the post-PPCI peak cTnI value minus the first admission cTnI value. STEMI was defined as continuous chest pain lasting $>30 \mathrm{~min}$, an elevated troponin I level, and an ECG finding of ST-segment elevation $>0.1 \mathrm{mV}$ in at least two contiguous leads or a new left bundle-branch block on an 18-lead ECG [10]. Hypertension was defined as a blood pressure $\geq 140 / 90 \mathrm{mmHg}$ in three occasions at rest or previous diagnosis of hypertension and current use of antihypertensive drugs. Diabetes mellitus (DM) was defined according to the $75 \mathrm{~g}$ oral glucose tolerance test (OGTT); that is, patients were diagnosed with DM if they met one of the following criteria: (i) a fasting plasma glucose level of $\geq 7.0 \mathrm{mmol} / \mathrm{L}$, (ii) a $2 \mathrm{~h}$ value of $\geq 11.1 \mathrm{mmol} / \mathrm{L}$ in $75 \mathrm{~g}$ OGTT, and (iii) a casual plasma glucose level of $\geq 11.1 \mathrm{mmol} / \mathrm{L}$. Dyslipidemia was defined by any of the following parameters: the total cholesterol (TC) $5.0 \mathrm{mmol} / \mathrm{L}$, low-density lipoprotein cholesterol (LDL-C) $\geq 3.0 \mathrm{mmol} / \mathrm{L}$, triglycerides (TG) $\geq 1.7 \mathrm{mmol} / \mathrm{L}$, high-density lipoprotein cholesterol (HLH-C) $\geq 1.2 \mathrm{mmol} / \mathrm{L}$ (women) or $\geq 1.0 \mathrm{mmol} / \mathrm{L}$ (men), or statin treatments. Height and weight were measured by trained medical staff; the body mass index was calculated by weight $(\mathrm{kg}) /$ height squared $\left(\mathrm{m}^{2}\right)$. The no-reflow phenomenon was defined as thrombolysis in a myocardial infarction (TIMI) flow grade $<3$ after PPCI.

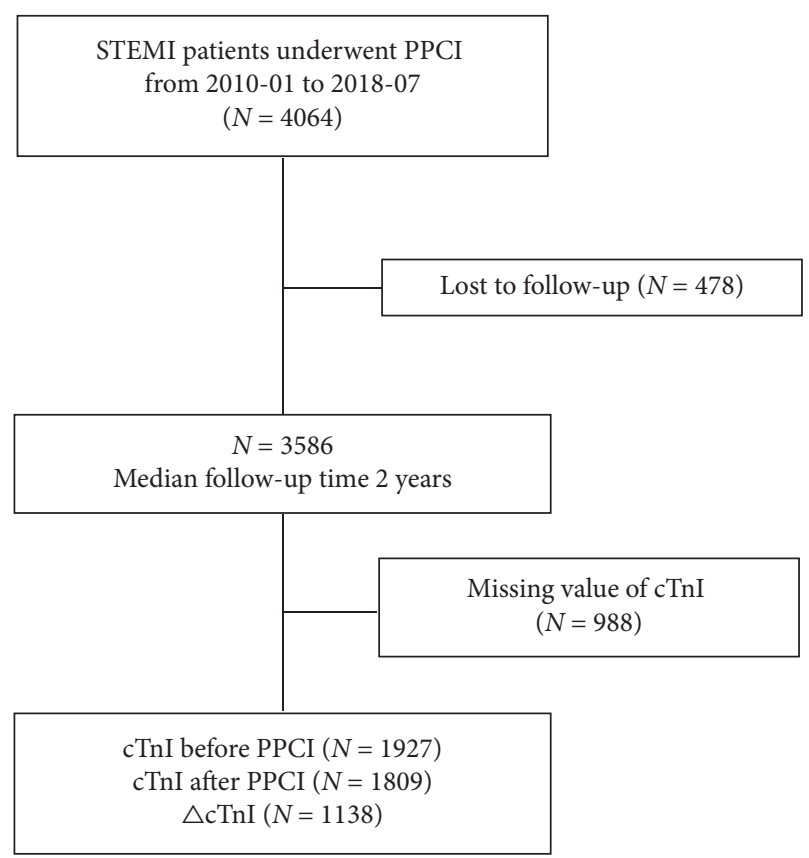

FIGURE 1: Flow chart of study enrollment. cTnI, cardiac troponin I; PPCI, primary percutaneous coronary intervention; STEMI, STsegment elevation myocardial infarction.

2.3. Biomarker Measurements. Blood samples were drawn from an antecubital vein in the morning after overnight fasting and collected into vacuum tubes containing EDTA for the measurement of plasma lipid and lipoprotein levels. TC, (HDL-C), LDL-C, TG, and homocysteine levels were analyzed by colorimetric enzymatic assays using an auto analyzer at the chemistry laboratory of the Fuwai Hospital Peking of Union Medical College. The level of C-reactive protein (CRP) was measured by biodirectional lateral flow immunoassay according to the procedure at the same chemistry laboratory.

Myocardial biomarkers were measured as follows: cTnI levels were measured on blood samples collected upon admission and after PPCI and serum was separated internally. The Abbott ARCHITECTi2000SR (Hong Kong, China) immunoassay system (batch number: 73099UI00) and Beckman UniCelDXI800 (California, USA) access immunoassay system (batch number: 624362) were used to analyze the cTnI level. The detection limit of the assay is $0.02 \mathrm{ng} / \mathrm{ml}$, and the decision limit for the diagnosis of MI is $0.07 \mathrm{ng} / \mathrm{ml}$.

2.4. Primary Stenting and Antiplatelet Therapy. PPCI (stenting/balloon dilatation/thrombus aspiration) was performed using standard criteria. Heparin or bivalirudin was used as periprocedural anticoagulant therapy. Glycoprotein IIb/IIIa inhibitors were used at the discretion of the operator. Commercially available stents were used. The dual antiplatelet therapy following PPCI consisted of oral aspirin (80-325 mg/ day continued indefinitely) and a P2Y12 inhibitor for at least 12 months. Other medications were prescribed at the discretion of the trained attending physicians. 
2.5. ECG and Echocardiography Collection. All ECG tracings from the emergency response teams and emergency department were collected and analyzed. Additional 18-lead ECGs were obtained upon arrival to the emergency department and twice daily thereafter until discharge. Each participant was scanned by trained ultrasonographers using the color Doppler ultrasonic diagnostic apparatus, cardiac ultrasonic measurement software, and a linear array transducer at a frequency of $12 \mathrm{MHz}$.

2.6. MACE and Follow-Up. For adverse events that occurred at follow-up, the following were evaluated: overall mortality, recurrence of MI (beyond $96 \mathrm{~h}$ of hospitalization, defined by the recurrence of chest pain accompanied by either re-STsegment elevation as described above or ST-segment depression attributed to myocardial ischemia and re-elevation of $\mathrm{c} T n \mathrm{I}>25 \%)$, and stroke.

2.7. Statistical Analysis. The normal distribution of outcome variables was confirmed by Kolmogorov-Smirnov tests. Baseline parameters and major adverse events during follow-up were presented as median (interquartile range) for continuous variables and as frequency and percentage for categorical variables. The relationship between baseline clinical, ECG, angiography characteristics, follow-up outcomes, and peak levels was assessed by the Spearman correlation. Survival analysis was performed with the Kaplan-Meier method. To assess the discrimination utility of cTnI, we plotted the time-dependent receiver operating characteristic (ROC) curves conducted by $\mathrm{R}$ language in order to obviate the limitation of potentially biased due to censoring. The predictive values of the ratio of delta $\mathrm{cTnI}$ to $\mathrm{cTnI}$ measured prior PPCI, delta cTnI, peak cTnI level post-PPCI, and the first admission cTnI level were obtained in the range of $0-1$ using the logistic regression model by controlling the following: history of hypertension, DM, hyperlipidemia, coronary artery bypass grafting, PCI, smoking, chest pain onset to hospital stay, Apo A, Apo B, CRP, TC, TG, HDLC, LDL-C, and medication history (aspirin, clopidogrel, warfarin, angiotensin-converting enzyme inhibitor, angiotensin receptor blockers, beta receptor blocker, statin, enzyme, and nitrates). The ROC curves were obtained by incorporating three predictive values. We tabulated the baseline characteristics of the cohort and, then, examined the bivariate association between these variables and $\triangle \mathrm{cTnI}$ quartiles. Differences across $\triangle \mathrm{cTnI}$ quartiles were evaluated by the analysis of variance (normally distributed variables) or Kruskal-Wallis test (skewed variables) for continuous variables and with the $\chi 2$ test for categorical variables. Univariable and multivariable Cox proportional hazards regression modeling was performed to characterize predictors of MACE. Categorical variables included the $\triangle \mathrm{c} T n I$ group, target lesion types of culprit vessels of MI, status of target organ thrombosis, status of the target organ occlusion, and whether the target lesion involves branches. Continuous variables included the $\triangle \mathrm{cTnI}$ level difference before and after PPCI and the GRACE score.
Significant variables analyzed were reported with their respective hazard ratios and $95 \%$ confidence limits. All $p$ values are two-tailed, and statistical significance was determined at $p<0.05$. Time-dependent ROC curves were performed with $\mathrm{R}$ language version $\mathrm{i} 386$ 3.6.2. The other analyses were performed with SPSS version 20.0 statistical software (SPSS, Inc., Chicago, IL).

\section{Results}

3.1. Patient Demographics. The median (interquartile range) time between symptom onsets to admission was $7 \mathrm{~h}$ $(8 \mathrm{~h})$. Patients' baseline clinical characteristics are summarized in Table 1. During the median 2-year follow-up, 293 patients (8.2\%) died (170) and other 68 patients had stroke. The mean age of the cohort was 59 years, and $75.5 \%$ were men (Table 1). Moreover, $53.1 \%$ of patients were current smokers. The prevalence rates of hypertension, hyperlipidemia, and DM were 59.6\%, 77.6\%, and 32.5\%, respectively. We compared the baseline characteristics of patients excluded from the analysis due to the lack of repeated troponin assessments versus patients included in the analysis in Table 2. There was no significant difference between two groups in the variables of the age, BMI, heart rate, systolic blood pressure, the history of PCI, history of CABG, the prevalence of hypertension, diabetes, the use of aspirin, warfarin, angiotensin receptor blocker, ezetimibe, and the incidence of stroke after follow-up. Figure 2 showed the time of the second sample taken after primary PCI. There were $509(44.728 \%)$ cases measured immediately after PPCI and 606 (53.251\%) measured during the index hospitalization. On the other hand, we found that $509(44.728 \%)$ cases measured immediately after PPCI and the cases measured during 0-7 days were 560 (49.209\%). Furthermore, we compared the peak troponin level with the delta troponin level and significant difference between two groups $(p<0.001)$.

3.2. Effects on Cardiac Troponin Elevation Kinetics. Correlation coefficients between cTnI and angiography characteristics, outcome at 2-year follow-up, and echocardiography measurement at discharge are shown in Table 3. Patients were categorized into three groups: cTnI levels upon admission (first cTnI), peak cTnI levels after PPCI (peak cTnI post-PPCI), and difference in cTnI between pre- and post-PPCI $(\triangle \mathrm{cTnI})$. Table 3 presents the independent significantly positive correlation between $\triangle \mathrm{cTnI}$ and type of the target lesion (coefficient $=0.091$, $p=0.005$ ), status of target organ thrombosis (coefficient $=0.154, \quad p \leq 0.001$ ), status of target organ with complete occlusion (coefficient $=0.203, p \leq 0.001$ ), left atrial diameter (LAD) on discharge (coefficient $=0.145$, $p \leq 0.001$ ), LVEDV on discharge (coefficient $=0.139$, $p=0.0050$ ), and mortality on the median 2 -year follow-up (coefficient $=0.438, p \leq 0.001)$. Moreover, an independent significantly negative correlation was found between $\triangle \mathrm{cTnI}$ and the time from symptom onset to admission (coefficient $=-0.161, p \leq 0.001$ ), TIMI flow grade pre-PPCI 
TABLE 1: Baseline characteristics of the study cohort.

\begin{tabular}{|c|c|c|c|c|}
\hline & All enrolled & $\begin{array}{l}\text { cTnI pre-PPCI } \\
\text { cohort }\end{array}$ & $\begin{array}{c}\text { Cohort of the maximum troponin in } \\
\text { measurement data }\end{array}$ & $\begin{array}{l}\triangle \mathrm{cTnI} \\
\text { cohort }\end{array}$ \\
\hline $\mathrm{N}$ & 3586 & 1927 & 1809 & 1138 \\
\hline Age (years) & $59(16)$ & $58(18)$ & $57(19)$ & $59(12.31)$ \\
\hline Male $(\%(n))$ & $75.7(2713)$ & $73.3(1412)$ & $72.2(1306)$ & $68.5(779)$ \\
\hline $\begin{array}{l}\text { Time from the onset of symptoms to } \\
\text { admission }(\mathrm{h})\end{array}$ & $7.00(8.00)$ & $7.00(9.00)$ & $7.00(8.00)$ & $7.00(9.00)$ \\
\hline $\begin{array}{l}\text { Time from the onset of symptoms to } \\
\text { balloon (h) }\end{array}$ & $\begin{array}{c}15.97 \\
(18.43)\end{array}$ & $15.58(23.90)$ & $14.86(25.58)$ & $14.38(20.46)$ \\
\hline BMI $\left(\mathrm{kg} / \mathrm{m}^{2}\right)$ & $25.76(4.48)$ & $25.26(5.10)$ & $25.16(5.46)$ & $25.99(3.76)$ \\
\hline Heart rate (beats per minute) & $76(19)$ & $75(18)$ & $75(18)$ & $77(36)$ \\
\hline Systolic blood pressure (mmHg) & $122(25)$ & $123(24)$ & $123(24)$ & $124(40)$ \\
\hline Diastolic blood pressure (mmHg) & $74(16)$ & $75(17)$ & $75(16.5)$ & $77(25)$ \\
\hline History of PCI $(\%(n))$ & $11.6(417)$ & $11.4(220)$ & $10.4(189)$ & $9.8(111)$ \\
\hline History of CABG $(\%(n))$ & $0.8(30)$ & $0.9(17)$ & $0.8(15)$ & $0.9(10)$ \\
\hline \multicolumn{5}{|l|}{ Risk factors } \\
\hline Hypertension $(\%(n))$ & $59.6(2136)$ & $59.1(1138)$ & $60.1(1088)$ & $59.3(675)$ \\
\hline Hyperlipidemia $(\%(n))$ & $77.6(2782)$ & $71.6(1380)$ & $71(1285)$ & $64.9(739)$ \\
\hline Smoking $(\%(n))$ & $53.1(1903)$ & $46.7(900)$ & $45.8(828)$ & $38.5(438)$ \\
\hline Diabetes $(\%(n))$ & $32.5(1165)$ & $33.1(638)$ & $31.9(577)$ & $32.2(366)$ \\
\hline \multicolumn{5}{|l|}{ Laboratory examinations } \\
\hline cTnI (ng/ml) & - & $0.37(3.00)$ & $4.16(14.73)$ & $3.71(15.78)$ \\
\hline Total cholesterol (mg/dl) & $2.40(3.31)$ & $2.97(3.09)$ & $3.07(3.33)$ & $1.37(1.04)$ \\
\hline HDL-cholesterol (mg/dl) & $1.12(3.94)$ & $1.87(3.35)$ & $1.26(3.22)$ & $3.15(1.82)$ \\
\hline LDL-cholesterol (mg/dl) & $2.30(3.06)$ & $2.42(1.8)$ & $2.60(1.34)$ & $1.43(1.28)$ \\
\hline Triglycerides $(\mathrm{mg} / \mathrm{dl})$ & $1.02(3.31)$ & $1.04(0.98)$ & $1.05(1.03)$ & $0.96(0.58)$ \\
\hline ALT (IU/L) & $\begin{array}{c}23.00 \\
(41.00)\end{array}$ & $28.00(41.10)$ & $33.00(42.93)$ & $25.44(5.62)$ \\
\hline AST (IU/L) & $\begin{array}{c}43.00 \\
(74.00)\end{array}$ & $55.00(78.00)$ & $55.00(25.00)$ & $26.00(41.00)$ \\
\hline TBil (ummol/L) & $\begin{array}{l}11.00 \\
(23.00)\end{array}$ & $14.00(24.00)$ & $15.00(4.90)$ & $29.00(60.00)$ \\
\hline D-BIL (ummol/L) & $1.50(3.70)$ & $2.27(5.10)$ & $2.60(4.90)$ & $13.00(21.58)$ \\
\hline ApoA $(g / L)$ & $1.21(3.52)$ & $1.25(3.23)$ & $1.37(4.09)$ & $7.43(88.34)$ \\
\hline ApoB $(g / L)$ & $1.08(0.38)$ & $1.05(0.35)$ & $1.04(0.38)$ & $1.40(4.49)$ \\
\hline \multicolumn{5}{|l|}{ Echocardiography data before PPCI } \\
\hline $\mathrm{LAD}(\mathrm{mm})^{\circ}$ & $35.00(5.00)$ & $35(5.00)$ & $35.00(5.00)$ & $35.00(5.00)$ \\
\hline IVSd (mm) & $10.00(1.00)$ & $10.00(8.00)$ & $10(1.00)$ & $7.00(8.00)$ \\
\hline LVEDV (mm) & $49(6.00)$ & $49.00(6.00)$ & $49.00(6.00)$ & $49.00(6.00)$ \\
\hline LVPWs (mm) & $9.00(1.00)$ & $9.00(1.00)$ & $9.00(1.00)$ & $9.00(1.00)$ \\
\hline $\mathrm{EF}(\%)$ & $\begin{array}{c}55.00 \\
(10.00)\end{array}$ & $56(0.00)$ & $55.00(42.92)$ & $56.00(10.00)$ \\
\hline \multicolumn{5}{|l|}{ Discharge medication regimen } \\
\hline Statin $(\%(n))$ & $81.5(2921)$ & $76.0(1465)$ & $72.2(1307)$ & $65.6(747)$ \\
\hline Aspirin $(\%(n))$ & $64.7(2321)$ & $76.8(1479)$ & $61.2(1107)$ & $67.0(762)$ \\
\hline Clopidogrel $(\%(n))$ & $66(2367)$ & $52.3(1007)$ & $54.9(993)$ & $42.9(488)$ \\
\hline Ticagrelor $(\%(n))$ & $20.1(721)$ & $27.9(537)$ & $21.9(397)$ & $27.0(307)$ \\
\hline Warfarin $(\%(n))$ & $0.4(15)$ & $0.3(6)$ & $0.4(8)$ & $0.2(2)$ \\
\hline ACEI $(\%(n))$ & $56.1(2011)$ & $54.0(1040)$ & $51.6(933)$ & $47.6(542)$ \\
\hline $\operatorname{ARB}(\%(n))$ & $6.7(242)$ & $6.3(122)$ & $6.7(122)$ & $5.9(67)$ \\
\hline Beta-blockers $(\%(n))$ & $76(2726)$ & $71.1(1370)$ & $67.6(1222)$ & $62.0(706)$ \\
\hline Ezetimibe $(\%(n))$ & $0.7(26)$ & $1.1(21)$ & $0.9(17)$ & $1.2(14)$ \\
\hline Diuretic $(\%(n))$ & $26(931)$ & $21.9(422)$ & $22.6(409)$ & $18.6(212)$ \\
\hline \multicolumn{5}{|l|}{ Endpoint events } \\
\hline MACE $(\%(n))$ & $8.2(293)$ & $4.9(94)$ & $5.9(107)$ & $4.1(47)$ \\
\hline Death $(\%(n))$ & $6(215)$ & $3.1(59)$ & $3.9(70)$ & $2.3(26)$ \\
\hline CV death $(\%(n))$ & $4.8(170)$ & $1.9(37)$ & $2.9(53)$ & $1.2(13)$ \\
\hline Stroke $(\%(n))$ & $1.9(68)$ & $1.3(26)$ & $1.7(31)$ & $1.5(17)$ \\
\hline
\end{tabular}

Continuous data are presented as mean \pm SD; categorical variables are presented as $\%(n)$. cTnI, cardiac troponin I; PPCI, primary percutaneous coronary intervention; BMI, body mass index; PCI, primary percutaneous coronary intervention; CABG, coronary artery bypass grafting; TC, total cholesterol; HDL-C, high-density lipoprotein cholesterol; LDL-C, low-density lipoprotein cholesterol; TG, triglyceride; ALT, alanine aminotransferase; AST, aspartate aminotransferase; TBIL, total bilirubin; D-BIL, direct bilirubin; ApoA, apolipoprotein A; ApoB, apolipoprotein B; LAD, left atrial diameter; IVSd, interventricular septal thickness diameter; LVEDV, left ventricular end systolic volume; LVPWs, left ventricular posterior wall thickness; EF, ejection fraction; ACEI, angiotensin-converting enzyme inhibitor; ARB, angiotensin receptor blocker; MACE, major adverse cardiovascular events; CV death, cardiovascular death. 
TABLE 2: Characteristics of the $\triangle \mathrm{cTnI}$ cohort versus cohort lack of repeated cTnI assessments.

\begin{tabular}{|c|c|c|c|}
\hline Variables & $\triangle \mathrm{cTnI}$ cohort & Cohort lack of repeated cTnI assessments & $p$ value \\
\hline $\mathrm{N}$ & 1138 & 2448 & 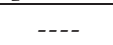 \\
\hline Age (years) & $59(12.31)$ & $59(12.08)$ & 0.095 \\
\hline Male $(\%(n))$ & $68.5(779)$ & $79.0(1934)$ & $\leq 0.001$ \\
\hline BMI $\left(\mathrm{kg} / \mathrm{m}^{2}\right)$ & $25.99(3.76)$ & $26.01(7.85)$ & 0.917 \\
\hline Heart rate (beats per minute) & $77.45(36.81)$ & $77.84(25.11)$ & 0.712 \\
\hline Systolic blood pressure $(\mathrm{mmHg})$ & $124(40)$ & $120(72)$ & 0.015 \\
\hline Diastolic blood pressure (mmHg) & $77(25)$ & $71(20)$ & $\leq 0.001$ \\
\hline History of PCI $(\%(n))$ & $9.8(111)$ & $12.5(306)$ & 0.019 \\
\hline History of CABG $(\%(n))$ & $0.9(10)$ & $0.8(20)$ & 0.845 \\
\hline Hypertension $(\%(n))$ & $59.3(675)$ & $59.7(1461)$ & 0.855 \\
\hline Hyperlipidemia $(\%(n))$ & $64.9(739)$ & $83.5(2043)$ & $\leq 0.001$ \\
\hline Smoking $(\%(n))$ & $38.5(438)$ & $59.9(1465)$ & $\leq 0.001$ \\
\hline Diabetes $(\%(n))$ & $32.2(366)$ & $32.6(799)$ & 0.561 \\
\hline Total cholesterol (mg/dl) & $1.37(1.04)$ & $2.54(2.05)$ & $\leq 0.001$ \\
\hline HDL-cholesterol (mg/dl) & $3.15(1.82)$ & $2.35(2.03)$ & $\leq 0.001$ \\
\hline LDL-cholesterol (mg/dl) & $1.43(1.28)$ & $2.35(2.03)$ & $\leq 0.001$ \\
\hline Triglycerides $(\mathrm{mg} / \mathrm{dl})$ & $0.96(0.58)$ & $1.19(0.97)$ & $\leq 0.001$ \\
\hline $\operatorname{ALT}(\mathrm{IU} / \mathrm{L})$ & $25.44(5.62)$ & $25.99(3.79)$ & 0.619 \\
\hline Statin $(\%(n))$ & $65.6(747)$ & $88.8(2174)$ & $\leq 0.001$ \\
\hline Aspirin $(\%(n))$ & $67.0(762)$ & 63.6(1559) & 0.060 \\
\hline Clopidogrel $(\%(n))$ & $42.9(488)$ & $76.8(1879)$ & $\leq 0.001$ \\
\hline Ticagrelor $(\%(n))$ & $27.0(307)$ & $16.9(414)$ & $\leq 0.001$ \\
\hline Warfarin $(\%(n))$ & $0.2(2)$ & $0.5(13)$ & 0.167 \\
\hline ACEI $(\%(n))$ & $47.6(542)$ & $60.0(1469)$ & $\leq 0.001$ \\
\hline $\operatorname{ARB}(\%(n))$ & $5.9(67)$ & $7.1(175)$ & 0.174 \\
\hline Beta-blockers $(\%(n))$ & $62.0(706)$ & $82.5(2020)$ & $\leq 0.001$ \\
\hline Ezetimibe $(\%(n))$ & $1.2(14)$ & $0.5(12)$ & 0.020 \\
\hline Diuretic $(\%(n))$ & $18.6(212)$ & $29.3(719)$ & $\leq 0.001$ \\
\hline $\operatorname{MACE}(\%(n))$ & $4.1(47)$ & $10.0(246)$ & $\leq 0.001$ \\
\hline Death $(\%(n))$ & $2.3(26)$ & $7.7(189)$ & $\leq 0.001$ \\
\hline CV death $(\%(n))$ & $1.2(13)$ & $6.4(157)$ & $\leq 0.001$ \\
\hline Stroke $(\%(n))$ & $1.5(17)$ & $2.1(51)$ & 0.292 \\
\hline
\end{tabular}

Continuous data are presented as mean \pm SD or median (interquartile range); categorical variables are presented as \% $(n)$. PCI, percutaneous coronary intervention; BMI, body mass index; CABG, coronary artery bypass grafting; TC, total cholesterol; HDL-C, high-density lipoprotein cholesterol; LDL-C, lowdensity lipoprotein cholesterol; TG, triglyceride; ALT, alanine aminotransferase; ACEI, angiotensin-converting enzyme inhibitor; ARB, angiotensin receptor blocker; MACE, major adverse cardiovascular events; CV death, cardiovascular death.

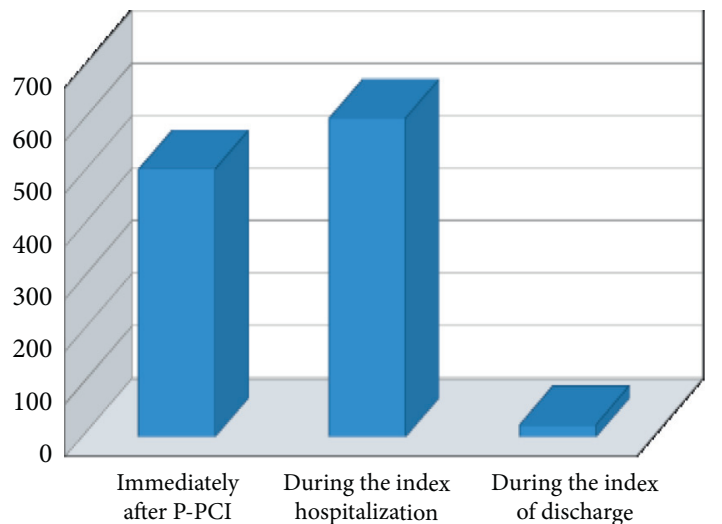

(a)

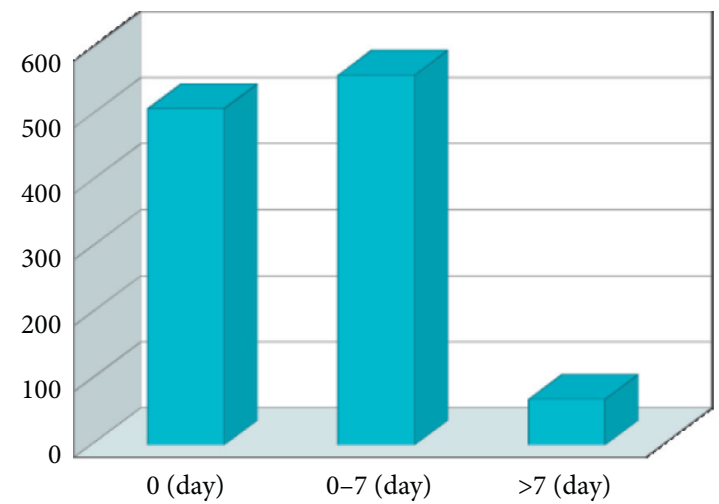

(b)

FIGURE 2: The time of the second sample taken after primary PCI. There were 509 (44.728\%) cases measured immediately after PPCI and 606 (53.251\%) measured during the index hospitalization. Five hundred and nine (44.728\%) cases were measured at the same day of primary PCI and the cases measured during 0-7 days were 560 (49.209\%).

(coefficient $=-0.214, p \leq 0.001$, minimum vessel diameter (coefficient $=-0.214, p \leq 0.001$ ), and LVEF on discharge (coefficient $=-0.019, p \leq 0.001)$.
3.3. Discrimination of the Value of the Ratio of Delta cTnI to cTnI Measured Prior PPCI, $\triangle c$ TnI, Peak cTnI after PPCI, and First cTnI. Figure 3 shows the survival (time-dependent) 
TABLE 3: Correlation analysis: associations between patient characteristics and cTnI on admission prior PPCI, peak troponin levels after PPCI, and the $\triangle \mathrm{cTnI}$ level difference before and after PPCI.

\begin{tabular}{|c|c|c|c|c|c|c|}
\hline & \multicolumn{2}{|c|}{ cTnI on admission prior PPCI } & \multicolumn{2}{|c|}{$\begin{array}{c}\text { Peak troponin levels after } \\
\text { PPCI }\end{array}$} & \multicolumn{2}{|c|}{$\begin{array}{l}\triangle c T n I \text { level difference before } \\
\text { and after PPCI }\end{array}$} \\
\hline & $\begin{array}{l}\text { Correlation } \\
\text { coefficient }\end{array}$ & $p$ value & $\begin{array}{l}\text { Correlation } \\
\text { coefficient }\end{array}$ & $p$ value & $\begin{array}{l}\text { Correlation } \\
\text { coefficient }\end{array}$ & $p$ value \\
\hline Time from symptom onset to admission & 0.432 & $<0.001^{*}$ & -0.004 & 0.880 & -0.161 & $<0.001^{*}$ \\
\hline TIMI flow grade prior PPCI & 0.046 & 0.059 & -0.206 & $\leq 0.001^{*}$ & -0.214 & $<0.001^{*}$ \\
\hline Minimum vessel diameter prior PPCI & 0.014 & 0.552 & -0.065 & $0.010^{*}$ & -0.214 & $<0.001^{*}$ \\
\hline The use of IABP & 0.036 & 0.136 & 0.025 & 0.313 & 0.056 & 0.081 \\
\hline GRACE score & 0.070 & $0.011^{*}$ & 0.046 & 0.148 & 0.017 & 0.637 \\
\hline Number of stents & -0.037 & 0.122 & $<0.001$ & 0.985 & -0.028 & 0.383 \\
\hline Target lesion types & -0.032 & 0.166 & 0.021 & 0.368 & 0.091 & $0.005^{*}$ \\
\hline The status of target organ thrombosis & -0.053 & $0.034^{*}$ & 0.160 & $<0.001^{*}$ & 0.154 & $<0.001^{*}$ \\
\hline $\begin{array}{l}\text { The target organ completed occlusion or } \\
\text { not }\end{array}$ & -0.068 & $0.005^{*}$ & 0.197 & $<0.001^{*}$ & 0.203 & $<0.001^{*}$ \\
\hline $\begin{array}{l}\text { The target lesion involves branches or } \\
\text { not }\end{array}$ & 0.063 & $0.009^{*}$ & 0.006 & 0.816 & -0.031 & 0.335 \\
\hline The target organ calcified or not & -0.034 & 0.165 & -0.031 & 0.224 & 0.025 & 0.446 \\
\hline Minimum vessel diameter after PPCI & -0.021 & 0.386 & 0.018 & 0.477 & 0.019 & 0.563 \\
\hline TIMI flow grade after PPCI & 0.026 & 0.280 & 0.050 & $0.048^{*}$ & -0.001 & 0.966 \\
\hline LAD on discharge & 0.051 & $0.046^{*}$ & 0.135 & $<0.001^{*}$ & 0.145 & $<0.001^{*}$ \\
\hline LVEDV on discharge & 0.067 & $0.007^{*}$ & 0.116 & $<0.001^{*}$ & 0.139 & $<0.001^{*}$ \\
\hline EF on discharge & -0.145 & $<0.001^{*}$ & -0.156 & $<0.001^{*}$ & -0.091 & $<0.001^{*}$ \\
\hline
\end{tabular}

cTnI, cardiac troponin I; PPCI, primary percutaneous coronary intervention; TIMI, thrombolysis in myocardial infarction; IABP, intra-aortic balloon pump; LAD, left atrial diameter; LVEDV, left ventricular end systolic volume; EF, ejection fraction.

ROC curves for the discrimination value of MACE of the ratio of delta cTnI to cTnI measured prior PPCI, $\triangle \mathrm{cTnI}$, peak cTnI after PPCI, and first cTnI. The areas under the ROC curve (AUC) are 0.730, 0.717, 0.590, and 0.546, respectively.

3.4. Stratified Analysis by the $\triangle c T n I$ Level Difference between Pre- and Post-PPCI. The characteristics of the group with the maximum difference (fourth group) in cTnI levels (ng/ $\mathrm{mL}$ ) that ranged from 19.161 to 101.66 (median value 52.00) at baseline, as shown in Table 4, are as follows. The group with the maximum $\triangle \mathrm{cTnI}$ level between $4.845 \mathrm{ng}$ / $\mathrm{ml}$ and $19.073 \mathrm{ng} / \mathrm{ml}$ was composed of patients with anterior wall myocardial infarction $(p<0.001)$, higher Global Registry of Acute Coronary Events (GRACE) score $(p=0.038)$, creatine kinase $\mathrm{MB}(p=0.023)$, and myohemoglobin $(p<0.001)$, while age, sex, the no-reflow phenomenon, triple-vessel disease, the number of stents, and risk factors including hypertension, hyperlipidemia, and DM failed to present statistical difference between groups (Table 4). Multivariate Cox regression analysis identified the $\triangle \mathrm{cTnI}$ level (HR: 1.018, 95\%, CI: 1.001-1.035, $p=0.042$ ), Q2 group (HR: 4.080, 95\% CI: 1.342-2.403, $p=0.013$ ), and uric acid (HR: $0.0=987,95 \% \mathrm{CI}$ : $0.977-0.988, p=0.018)$ as relevant factors for MACE during follow-up (Table 5).

3.5. Survival Analysis of the $\triangle c T n I$ Level Difference before and after PPCI. The median follow-up time was 2 (range $<1-8.35$ ) years. The Kaplan-Meier curves depicted a cumulative probability of MACE, mortality, recurrent myocardial infarction, and angina pectoris for patients stratified into quartiles of the $\triangle \mathrm{c} T$ nI level at enrollment (log rank $p=0.035,0.049,0.015,0.026$ ) (Figure 4 ).

\section{Discussion}

This study investigated defined variables in a large-scale, well-described, and contemporary-treated STEMI population from China. The novelty of the study was the assessment of the discrimination value of the $\triangle c T n I$ and the ratio of $\triangle c T n I$ to $c T n I$ on initial admission to the incidence of MACE which remains largely unknown. The researchers followed strict inclusion and exclusion criteria, which facilitated a reasonably streamlined and comparable hospital flow for all subjects. On the other hand, the use of timedependent ROC curves may obviate potentially biased due to censoring.

4.1. Effects on High $\triangle c T n I$ Kinetics. Several prior investigations supported that the early release of cTnI following AMI is caused by the washout mechanism from the infarcted zone into the serum, a process which is facilitated by early restoration of blood flow to the infarcted tissue [11-13]. In this study, the analyzed patients were selected from a large retrospective cohort of 3586 patients with STEMI undergoing contemporary primary percutaneous revascularization and supported the independent significantly positive correlation between $\triangle \mathrm{cTnI}$ and the type of the target lesion, status of target organ thrombosis, status of target organ with complete occlusion, LAD on discharge, LVEDV on discharge, and mortality on the median 2-year follow-up. Several previous studies [14-18] have shown that cardiac 


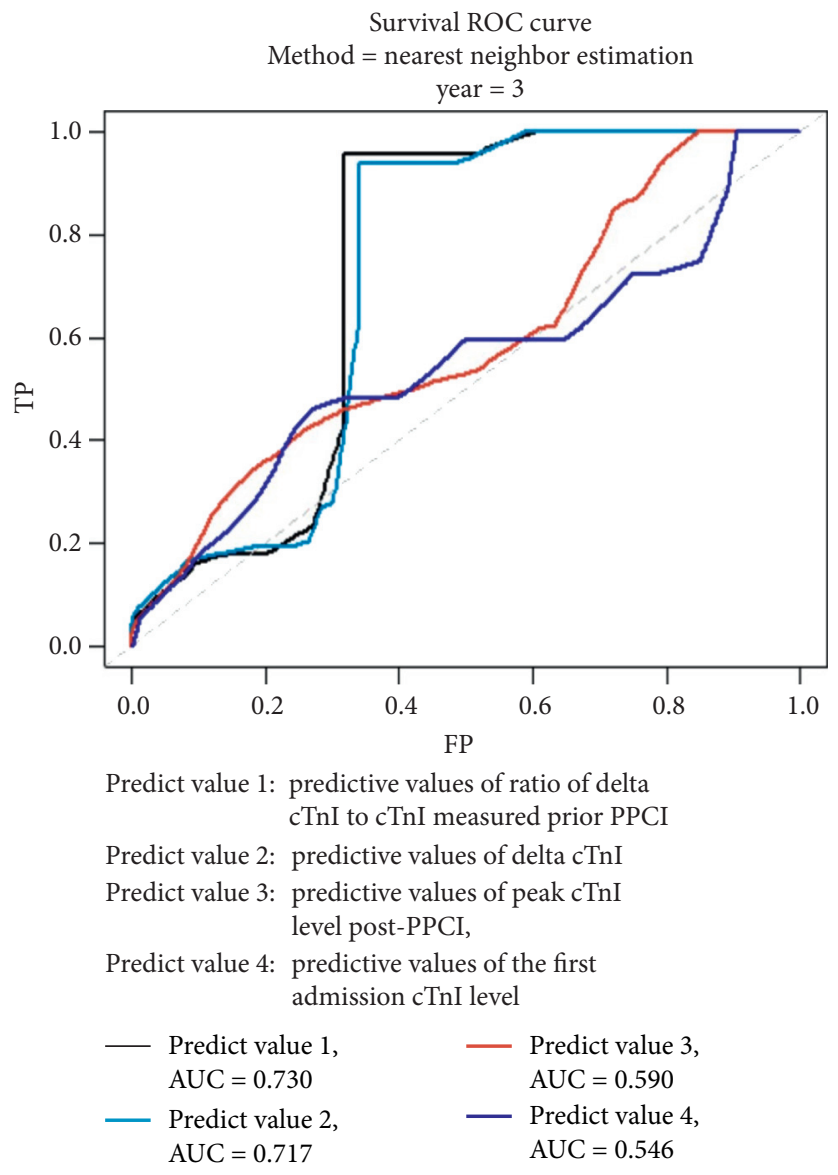

FIgURE 3: Survival ROC curve. Predictive value 1: predictive values of ratio of delta cTnI to cTnI measured prior PPCI; predictive value 2: predictive values of delta cTnI; predictive value 3: predictive values of the peak cTnI level after PPCI; and predictive value 4: predictive values of the first admission cTnI level. Survival receiver operating characteristic curves for a model including the ratio of delta cTnI to cTnI measured prior PPCI (black line), $\triangle \mathrm{cTnI}$ level (wathet blue line), peak level after PPCI (red line), and cTnI concentrations at admission (dark blue line). The areas under the ROC curve are shown for the graph. AUC, area under the curve; ROC, survival receiver operating characteristic; TP, true positive; and FP, false positive.

TABLE 4: Analysis stratified according to the quartiles of delta troponin I.

\begin{tabular}{|c|c|c|c|c|c|}
\hline Variables & $\begin{array}{c}\text { Quartile I } \\
(n=284) \\
-79.724-0.347\end{array}$ & $\begin{array}{c}\text { Quartile II } \\
(n=284) \\
0.350-4.708\end{array}$ & $\begin{array}{c}\text { Quartile III } \\
(n=285) \\
4.845-19.073\end{array}$ & $\begin{array}{c}\text { Quartile IV } \\
\quad(n=285) \\
19.161-101.660\end{array}$ & $p$ value \\
\hline Median of the $\triangle \mathrm{c}$ TnI level $(\mathrm{ng} / \mathrm{ml})$ & -5.14 & 2.09 & 10.38 & 52.00 & - \\
\hline Age (years) & $60(17)$ & $59(15)$ & $57(15)$ & $59(16)$ & 0.192 \\
\hline Male $(\%(n))$ & $77.1(219)$ & $79.6(226)$ & $82.5(235)$ & $83.9(239)$ & 0.073 \\
\hline BMI $\left(\mathrm{kg} / \mathrm{m}^{2}\right)$ & $25.25(4.65)$ & 25.95(4.59) & $25.90(4.47)$ & $25.95(4.38)$ & 0.284 \\
\hline Heart rate (beats per minute) & $75.00(18.00)$ & $74.00(15.00)$ & $73.00(18.00)$ & $76.00(19.00)$ & 0.840 \\
\hline Systolic blood pressure (mmHg) & $126.00(22.00)$ & $124.00(17.00)$ & $123.00(23.00)$ & $122.00(25.00)$ & 0.670 \\
\hline Diastolic blood pressure $(\mathrm{mmHg})$ & $77.00(17.00)$ & $76.00(15.25)$ & $77.00(17.00)$ & $77.00(17.00)$ & 0.534 \\
\hline Anterior wall myocardial infarction & 140 & 118 & 99 & 143 & $<0.001^{*}$ \\
\hline History of PCI $(\%(n))$ & $9.9(28)$ & $12.7(36)$ & $9.8(28)$ & $6.7(19)$ & 0.120 \\
\hline History of CABG $(\%(n))$ & $0.7(2)$ & $0.4(1)$ & $0.7(2)$ & $1.8(5)$ & 0.307 \\
\hline GRACE score & $102(40)$ & $103(36)$ & $95(34)$ & $104(36)$ & $0.038^{*}$ \\
\hline $\begin{array}{l}\text { The time from chest pain to vessel } \\
\text { reperfusion (day) }\end{array}$ & $0.47(0.60)$ & $0.35(0.50)$ & $0.32(0.34)$ & $0.31(0.33)$ & $<0.001^{*}$ \\
\hline \multicolumn{6}{|l|}{ Risk factors } \\
\hline Hypertension $(\%(n))$ & $59.5(169)$ & $59.5(169)$ & $55.1(157)$ & $63.2(180)$ & 0.277 \\
\hline Hyperlipidemia $(\%(n))$ & $77.8(221)$ & $75.4(214)$ & $63.2(180)$ & $43.5(124)$ & 0.159 \\
\hline
\end{tabular}


TABLE 4: Continued.

\begin{tabular}{|c|c|c|c|c|c|}
\hline Variables & $\begin{array}{c}\text { Quartile I } \\
(n=284) \\
-79.724-0.347\end{array}$ & $\begin{array}{c}\text { Quartile II } \\
(n=284) \\
0.350-4.708\end{array}$ & $\begin{array}{c}\text { Quartile III } \\
\quad(n=285) \\
4.845-19.073\end{array}$ & $\begin{array}{c}\text { Quartile IV } \\
\quad(n=285) \\
19.161-101.660\end{array}$ & $p$ value \\
\hline Smoking $(\%(n))$ & $56.3(160)$ & $44(125)$ & $36.1(103)$ & $17.2(49)$ & 0.848 \\
\hline Diabetes $(\%(n))$ & $32.7(93)$ & $35.3(100)$ & $30.9(88)$ & $29.8(85)$ & 0.536 \\
\hline \multicolumn{6}{|l|}{ Laboratory examinations } \\
\hline MYO & $20.00(72.16)$ & $20.99(66.83)$ & $47.16(229.70)$ & $201.68(831.70)$ & $<0.001^{*}$ \\
\hline CK-MB (ng/mL) & $51.50(540.15)$ & $25.00(161.30)$ & $56.00(189.00)$ & $42.04(246.54)$ & 0.023 \\
\hline NT-proBNP (pg/ml) & $56.39(684.29)$ & $183.60(1170)$ & $148.90(755.28)$ & $111.7(965.25)$ & 0.514 \\
\hline $\mathrm{CRP}(\mathrm{mg} / \mathrm{L})$ & $6.70(8.86)$ & $4.83(8.45)$ & $5.29(9.00)$ & $6.51(8.06)$ & 0.085 \\
\hline Uric acid $(\mathrm{mmol} / \mathrm{L})$ & $8.39(69.46)$ & $5.55(70.52)$ & $5.75(68.26)$ & $4.68(7.67)$ & $<0.001^{*}$ \\
\hline Creatinine (ummol/L) & $1.42(4.36)$ & $2.05(4.43)$ & $1.45(1.32)$ & $2.07(0.91)$ & $<0.001^{*}$ \\
\hline \multicolumn{6}{|l|}{ Echocardiography data pre-PPCI } \\
\hline $\mathrm{LAD}(\mathrm{mm})$ & $35.00(4.00)$ & $35.00(5.00)$ & $35.50(5.00)$ & $36.00(5.00)$ & 0.310 \\
\hline IVSd (mm) & $9.00(2.00)$ & $9.00(2.00)$ & $9.00(2.00)$ & $9.00(2.00)$ & 0.476 \\
\hline LVEDV (mm) & $48.00(5.00)$ & $49.00(6.00)$ & $49.00(6.00)$ & $50.00(6.00)$ & $0.007^{*}$ \\
\hline LVPWs (mm) & $10.00(1.00)$ & $9.00(1.00)$ & $9.00(1.00)$ & $9.00(1.00)$ & 0.012 \\
\hline $\mathrm{EF}(\%)$ & $56.00(10.00)$ & $56.00(9.00)$ & $57.00(7.00)$ & $54.00(10.00)$ & $<0.001^{*}$ \\
\hline \multicolumn{6}{|l|}{ Angiographic findings pre-PPCI } \\
\hline Triple-vessel disease $(\%(n))$ & $34.2(97)$ & $32.4(92)$ & $33.3(95)$ & $33.0(94)$ & 0.984 \\
\hline Length of target lesions (mm) & $24.00(17.00)$ & $24.00(17.00)$ & $25.00(16.25)$ & $23.5(16.00)$ & 0.479 \\
\hline Target lesion types $=C(\%(n))$ & $59.5(169)$ & $62.7(178)$ & $65.3(186)$ & $69.5(198)$ & 0.096 \\
\hline The status of target organ thrombosis $(\%(n))$ & $38.7(110)$ & $44.3(126)$ & $57.9(165)$ & $57.9(165)$ & $0.002^{*}$ \\
\hline The target organ completed occlusion $(\%(n))$ & $48.6(138)$ & $54.9(156)$ & $69.8(199)$ & $73.3(209)$ & $<0.001^{*}$ \\
\hline The target lesion involves branches $(\%(n))$ & $37.3(106)$ & $30.6(87)$ & $31.6(90)$ & $27.1(77)$ & 0.150 \\
\hline The target organ calcification $(\%(n))$ & $44.0(125)$ & $44.0(125)$ & $42.2(120)$ & $43.6(124)$ & 0.957 \\
\hline TIMI flow grade prior PPCI $=0(\%(n))$ & $51.4(146)$ & $60.2(171)$ & $73.0(208)$ & $77.2(220)$ & $<0.001^{*}$ \\
\hline Minimum vessel diameter prior PPCI $(\mathrm{mm})$ & $0.00(0.175)$ & $0.00(0.15)$ & $0.00(0.035)$ & $0.00(0.00)$ & $<0.001^{*}$ \\
\hline \multicolumn{6}{|l|}{ Procedural data } \\
\hline The use of IABP $(\%(n))$ & $6.0(17)$ & $3.2(9)$ & $4.2(12)$ & $6.7(19)$ & 0.100 \\
\hline Number of cardiac stents $>1(\%(n))$ & $26.8(76)$ & $21.9(62)$ & $26.6(67)$ & $23.6(67)$ & 0.272 \\
\hline No-reflow phenomenon $(\%(n))$ & $2.8(8)$ & $3.6(10)$ & $3.2(9)$ & $3.2(9)$ & 0.377 \\
\hline Minimum vessel diameter after PPCI (mm) & $3.00(1.00)$ & $3.00(1.00)$ & $3.00(1.00)$ & $3.00(1.00)$ & 0.949 \\
\hline \multicolumn{6}{|l|}{ Discharge medication regimen } \\
\hline Statin $(\%(n))$ & $86.3(245)$ & $75.0(213)$ & $66.0(188)$ & $35.4(101)$ & $0.009^{*}$ \\
\hline Aspirin $(\%(n))$ & $88.7(252)$ & $77.8(221)$ & $66.3(189)$ & $35.1(100)$ & $<0.001^{*}$ \\
\hline Clopidogrel $(\%(n))$ & $59.9(170)$ & $52.1(148)$ & $38.2(109)$ & $21.4(61)$ & $0.028^{*}$ \\
\hline Ticagrelor $(\%(n))$ & $33.1(94)$ & $29.9(85)$ & $29.5(84)$ & $15.4(44)$ & 0.445 \\
\hline ACEI $(\%(n))$ & $63.7(181)$ & $57.7(164)$ & $46.3(132)$ & $22.8(65)$ & $0.023^{*}$ \\
\hline $\operatorname{ARB}(\%(n))$ & $7.4(21)$ & $5.6(16)$ & $5.6(16)$ & $4.9(14)$ & 0.468 \\
\hline Beta-blockers $(\%(n))$ & $83.1(236)$ & $73.2(208)$ & $58.9(168)$ & $33.0(94)$ & $0.024^{*}$ \\
\hline Ezetimibe $(\%(n))$ & $2.5(7)$ & $0.7(2)$ & $1.4(4)$ & $0.4(1)$ & 0.370 \\
\hline Nitrate $(\%(n))$ & $75.4(214)$ & $63.7(181)$ & $52.3(149)$ & $26.7(76)$ & $0.002^{*}$ \\
\hline Diuretic $(\%(n))$ & $26.8(76)$ & $20.1(57)$ & $15.4(44)$ & $12.3(35)$ & 0.300 \\
\hline \multicolumn{6}{|c|}{ Clinical outcomes evaluated during the follow-up } \\
\hline MACE $(\%(n))$ & $2.1(6)$ & $6.7(19)$ & $3.9(11)$ & $3.9(11)$ & 0.052 \\
\hline Death $(\%(n))$ & $1.4(4)$ & $2.8(8)$ & $1.8(5)$ & $3.2(9)$ & 0.342 \\
\hline $\mathrm{CV}$ death $(\%(n))$ & $0.8(2)$ & $2.2(6)$ & $0.7(2)$ & $1.1(3)$ & 0.579 \\
\hline Recurrent myocardial infarction & $1.1(3)$ & $2.8(8)$ & $2.1(6)$ & $2.1(6)$ & 0.225 \\
\hline Angina pectoris & $5.7(16)$ & $7.1(20)$ & $7.4(21)$ & $3.9(11)$ & 0.575 \\
\hline Stroke $(\%(n))$ & $1.4(4)$ & $2.1(6)$ & $1.4(4)$ & $1.1(3)$ & 0.893 \\
\hline
\end{tabular}

Continuous data are presented as mean \pm SD or median (interquartile range); categorical variables are presented as \% (n). cTnI, cardiac troponin I; BMI, body mass index; PCI, primary percutaneous coronary intervention; CABG, coronary artery bypass grafting; TC, total cholesterol; HDL-C, high-density lipoprotein cholesterol; LDL-C, low-density lipoprotein cholesterol; TG, triglyceride; ALT, alanine aminotransferase; AST, aspartate aminotransferase; TBIL, total bilirubin; D-BIL, direct bilirubin; ApoA, apolipoprotein A; ApoB, apolipoprotein B; LAD, left atrial diameter; IVSd, interventricular septal thickness diameter; LVEDV, left ventricular end systolic volume; LVPWs, left ventricular posterior wall thickness; EF, ejection fraction; ACEI, angiotensin-converting enzyme inhibitor; $\mathrm{ARB}$, angiotensin receptor blocker. 
TABLE 5: Results of the univariate and multivariable Cox proportional hazards model applied to assess correlates of 2-year MACE.

\begin{tabular}{|c|c|c|c|c|}
\hline \multirow{2}{*}{ Baseline parameters } & \multicolumn{2}{|c|}{ Univariate Cox regression } & \multicolumn{2}{|c|}{ Multivariate Cox regression } \\
\hline & HR (95\% CI) & $p$ value & HR (95\% CI) & $p$ value \\
\hline$\triangle \mathrm{cTnI}$ & $1.013(1.004-1.022)$ & $0.005^{*}$ & $1.018(1.001-1.035)$ & $0.042^{*}$ \\
\hline $\mathrm{Q} 1 \triangle \mathrm{cTnI}$ & Ref & Ref & Ref & Ref \\
\hline $\mathrm{Q} 2 \Delta \mathrm{cTnI}$ & $3.399(1.357-8.514)$ & $0.009^{*}$ & $4.080(1.342-12.403)$ & $0.013^{*}$ \\
\hline $\mathrm{Q} 3 \triangle \mathrm{cTnI}$ & $2.637(0.971-7.162)$ & 0.057 & $2.561(0.744-8.819)$ & 0.136 \\
\hline $\mathrm{Q} 4 \Delta \mathrm{cTnI}$ & $3.583(1.317-9.752)$ & $0.012^{*}$ & $1.566(0.290-8.444)$ & 0.602 \\
\hline BMI & $0.951(0.876-1.033)$ & 0.234 & $0.945(0.863-1.035)$ & 0.223 \\
\hline Anterior wall myocardial infarction & $1.255(0.709-2.224)$ & 0.436 & $1.315(0.681-2.540)$ & 0.414 \\
\hline Uric acid & $0.984(0.974-0.994)$ & $0.002^{*}$ & $0.987(0.977-0.998)$ & $0.018^{*}$ \\
\hline Number of stents & $0.776(0.477-1.261)$ & 0.306 & $1.152(0.697-1.905)$ & 0.581 \\
\hline No-reflow phenomenon & $1.014(0.139-7.387)$ & 0.989 & $1.463(0.190-11.259)$ & 0.715 \\
\hline \multicolumn{5}{|l|}{ Target lesion types } \\
\hline A & Ref & Ref & Ref & Ref \\
\hline B1 & $0.272(0.091-0.813)$ & $0.020^{*}$ & - & 0.908 \\
\hline $\mathrm{B} 2$ & $0.103(0.031-0.340)$ & $<0.001^{*}$ & - & 0.917 \\
\hline $\mathrm{C}$ & $0.162(0.067-0.393)$ & $<0.001^{*}$ & - & 0.915 \\
\hline Organ thrombosis & $1.011(0.622-1.643)$ & 0.965 & $0.998(0.481-2.075)$ & 0.988 \\
\hline Completed occlusion & $0.990(0.524-1.872)$ & 0.977 & $0.864(0.411-1.820)$ & 0.701 \\
\hline Target lesion involving branches & $0.930(0.481-1.797)$ & 0.829 & $0.943(0.456-1.952)$ & 0.875 \\
\hline
\end{tabular}

MACE, major adverse cardiovascular events; HR, hazard ratio; cTnI, cardiac troponin I; PPCI, primary percutaneous coronary intervention; BMI, body mass index.

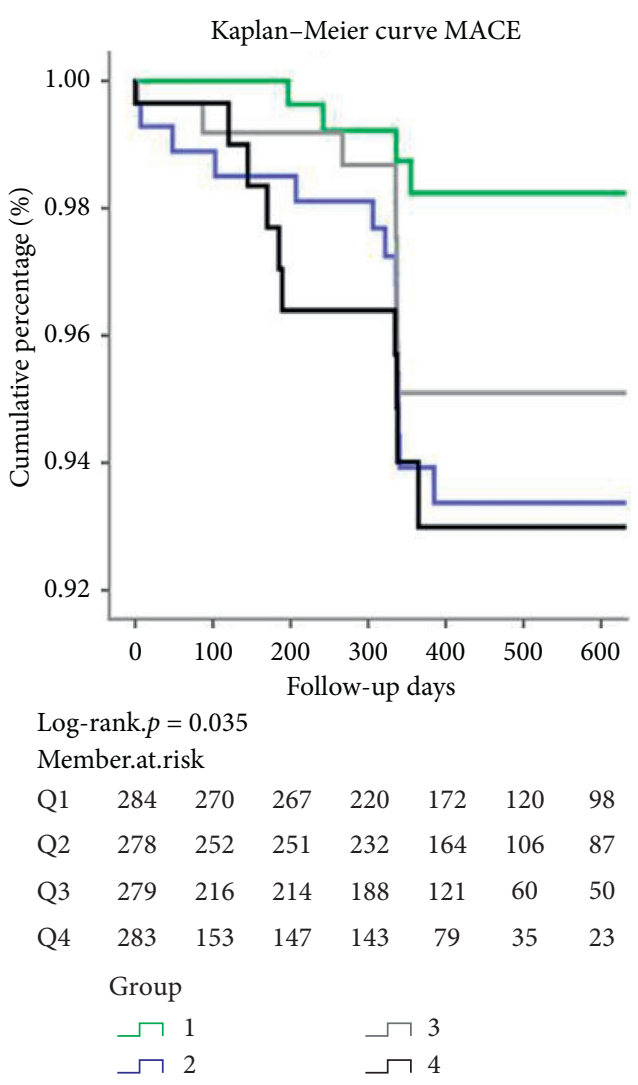

(a)

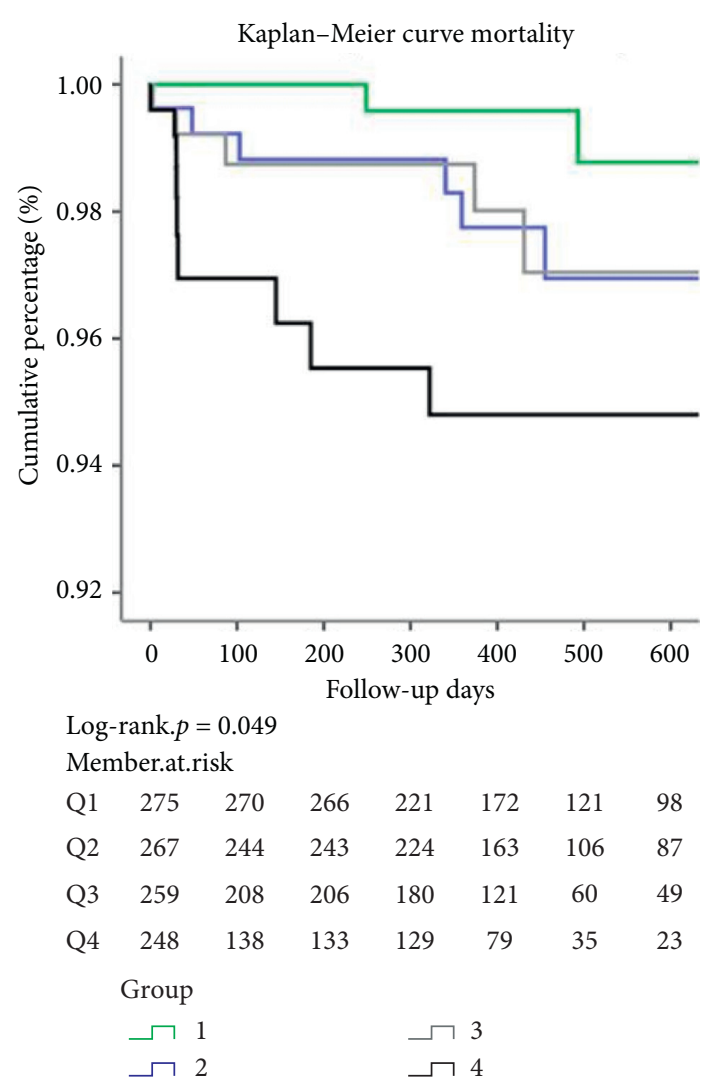

(b)

Figure 4: Continued. 


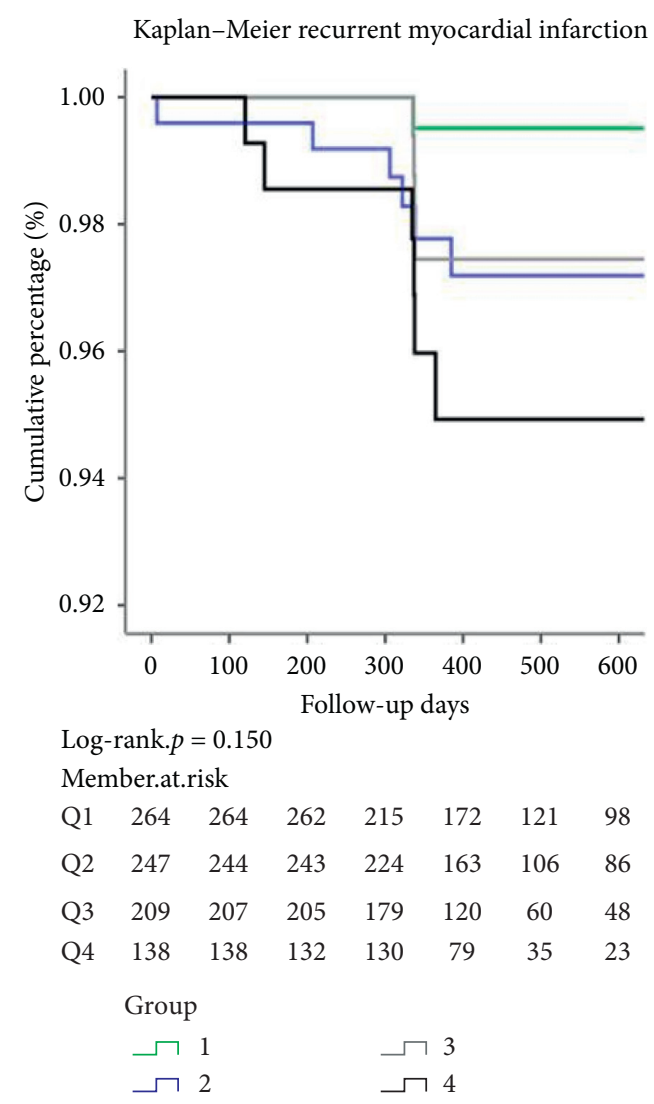

(c)

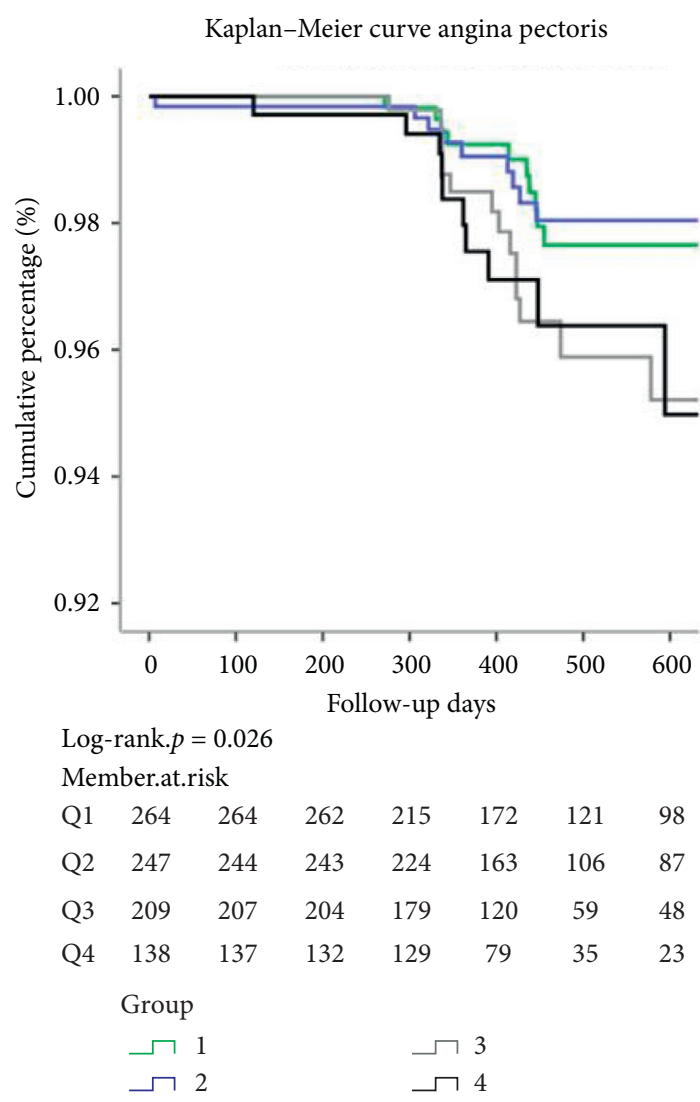

(d)

FIGURE 4: Cumulative incidence of myocardial infarction or cardiac death in patients with troponin concentrations. Patients were stratified into four groups based on the $\triangle \mathrm{cTn}$ I level. Q1: first quartile (green line); Q2: second quartile (blue line); Q3: third quartile (grey line); and Q4: fourth quartile (black line). (a) Kaplan-Meier curves depicting the cumulative probability for MACE (log rank $p=0.035$ ). (b) Kaplan-Meier curves depicting the cumulative probability for mortality (log rank $p=0.049$ ). (c) Kaplan-Meier curves depicting the cumulative probability for recurrent myocardial infarction ( $\log$ rank $p=0.150$ ). (d) Kaplan-Meier curves depicting the cumulative probability for angina pectoris ( $\log \operatorname{rank} p=0.026)$. MACE: major adverse cardiovascular events.

troponin could predict the LV function after STEMI patients were reperfused pharmacologically. Moreover, we found that peak troponin concentration after PPCI was associated with the TIMI flow grade at pre-PPCI (coefficient $=-0.206$, $p \leq 0.001$ ), minimum vessel diameter prior PPCI (coefficient $=-0.065, \quad p=0.010$ ), LAD on discharge (coefficient $=0.135, \quad p \leq 0.001$ ), LVEDV on discharge (coefficient $=0.116, \quad p \leq 0.001$ ), and LVEF on discharge (coefficient $=-0.156, p \leq 0.001$ ).

4.2. $\triangle c T n I$ and the Ratio of $\triangle c T n$ Have a Discriminative Value of Follow-Up Outcomes. Many studies reported [19-22] that an increased first cTnI level and peak cTnI were associated with an adverse outcome of primary angioplasty in AMI. Matetzky et al. [23] found that, in AMI patients with ST-segment elevation, an elevated cTnI on admission was associated with an increased risk of primary angioplasty failure and a more complicated clinical course. Testa [24] reported that a small increase in troponin concentration after a successful elective PCI was not infrequent and did not affect the outcome. Our study focused on the relationship between $\triangle \mathrm{cTnI}$ between post-PPCI peak cTnI and first cTnI and comprehensive 2-year outcome assessments in PPCI-treated STEMI patients, which are an important addition to the current knowledge, as similar studies on the era remain limited. We found that the group with the maximum $\triangle \mathrm{c}$ TnI level between 4.845 and 19.073 (median 52.00) has a statistically higher number of patients with target lesion thrombosis $(p=0.002)$, complete occlusion of the target lesion $(p \leq 0.001)$, TIMI blood flow grade 0 at prePPCI $(p \leq 0.001)$, and statistically significant lower use of cardiovascular-related drugs at post-PPCI than other groups. Based on the Kaplan-Meier analysis of the probability of death, the mortality rate of the group with the maximum $\triangle \mathrm{cTnI}$ (median 52.00) was significantly higher than that of other groups.

4.3. Characteristics of the Group with the Maximum $\triangle c T n I$ Level. Sezer et al. [25] reported that, in patients with anterior wall AMI treated with PPCI, absolute and relative neutrophilia and mean platelet volume were independently associated with impaired microvascular perfusion. Kobayashi et al. [26] observed that a wraparound LAD predicted adverse clinical outcomes (hazard ratio: 2.18, $p=0.02$ ) and severe heart failure (odds ratio 3.31, $p=0.049$ ) 
at 3 years in patients with anterior STEMI who underwent PPCI. These observations, together with the present results, tend to support the characteristics of the fourth group of the $\triangle c T n I$ level with the maximum cases of anterior wall MI and higher risk for MACE. The pathophysiology of the no-reflow phenomenon is complex, and a series of consistent data has $[27,28]$ clearly shown that the no-reflow phenomenon has a strong negative effect on the outcome. In a population-based global registry, the NCDR study reported [29] that the noreflow phenomenon is associated with an increased risk of adverse postprocedure hospital course including higher inhospitality mortality $(6.8 \%$ vs. $2.9 \%$; $p=0.01)$, cerebrovascular accident $(1.5 \%$ vs. $0 \% ; p<0.001)$, postprocedure bleeding $(2.3 \%$ vs. $0.5 \% ; p=0.009)$, and cardiogenic shock (3.8\% vs. $1.2 \% ; p=0.011)$. The no-reflow phenomenon is a process in which prolonged ischemia caused changes in endothelial cells, and optimal treatment of hyperglycemia is a significant target in preventing $[30,31]$. On the contrary, we did not find a significant statistical difference in the noreflow phenomenon between the four groups according to $\triangle c T n I$ at pre- and post-PPCI $(p=0.337)$. This was possibly caused by the number of participants and the rare occurrence of the no-reflow phenomenon after PPCI in our study.

The findings from studies $[32,33]$ emphasized the counterbalancing effects of ischemic and hemorrhagic complications after stent implantation and revealed the significant effect of the stent on patient outcomes. The analysis of the characteristics of the fourth group with poor prognosis of MACE failed to determine that the number of stents $(p=0.272)$ and triple-vessel disease $(p=0.984)$ have a significant statistical difference with other groups. Because of the inadequate calibration, the actual prevalence of the four groups according to the $\triangle c T n I$ level fluctuation may need to be viewed with caution in Chinese population with STEMI following PPCI. Several studies have reported the predictive accuracy of the GRACE risk score in different patient populations. A study of contemporary populations [34] with a validated GRACE risk score suggested that the GRACE risk score could predict in-hospital and 6-month mortality and has high collinearity between LVEF in a cohort of patients with ACS. The study [35] concluded that the GRACE risk score demonstrated a significant discriminatory ability for adverse outcomes. In conclusion, these results indirectly support that the statistically significant higher GRACE risk score in the fourth $\triangle \mathrm{cTnI}$ group has contributed to the higher incidence of MACE $(p \leq 0.001)$. Furthermore, mathematical models and artificial intelligence have recently come in help in the setting, and integration into the clinical workflow might significantly improve patient outcomes [36]. The novel dynamical model synthetically describes the basic mechanisms underlying cTnI release into the plasma after the onset of AMI which provide the clinicians with a quantitative tool to analyze the series.

4.4. Limitations. Nevertheless, this study has several potential limitations. Firstly, it is a single-center, retrospective study design with strict inclusion criteria, and many patients who did not have a valid cTnI result were excluded, which resulted in selection bias. Secondly, patients have been enrolled during a long span of time, which could bring about a confounding effect due to the improvements of interventional techniques and progress in medication. Multicenter studies with a larger sample of patients admitted in a short period would be preferred to validate the results of this study. While the discrepancies in discharge medication were explored, a potentially unbalanced distribution of missing values may have influenced the results. Finally, we did not analyze some acute phase biomarkers such as ST-segment recovery and reperfusion ventricular arrhythmia "bursts" which are also related to the outcome in our models. Thus, the relative predictive information provided by $\triangle \mathrm{c} T n I$ simultaneously considered with such additional biomarkers remains an important area for future research.

\section{Conclusions}

The main findings of this study are as follows: (1) $\triangle \mathrm{cTnI}$ and the ratio of $\triangle \mathrm{cTnI}$ to $\mathrm{cTnI}$ on initial admission were significant prognostic indicators in patients with MACE compared with first cTnI and peak cTnI after PPCI. (2) The maximum $\triangle c T n I$ level (median 52.00) was associated with a higher incidence of MACE, mortality, and angina pectoris at media 2-year follow-up than the other groups. (3) A higher number of patients with the maximum $\triangle c$ TnI level more likely had anterior wall MI $(p<0.001)$, a higher GRACE score $(p=0.038)$, myohemoglobin $(p<0.001)$, and TIMI flow grade 0 at pre-PPCI $(p<0.001)$.

Our findings supported that repeated measurements of $c T n I$ and $\triangle c T n I$ could provide significant incremental information for risk stratification of STEMI patients who underwent PPCI. It represents a valuable, inexpensive, and readily available tool for assessment of risk, especially of long-term MACE; in addition, our results suggest that routine use of $\triangle \mathrm{cTnI}$ values provide complementary information to well-established clinical risk factors. Furthermore, one can envision a $\triangle \mathrm{cTn} I$ biomarker-guided approach to implement aggressive medical treatment strategies before discharge in patients at increased risk. Finally, it could provide help in the future selection of individuals considered for inclusion in clinical trials aimed at improving outcomes in such a population.

\section{Data Availability}

The datasets uesd and/or analyzed during this study are available from the corresponding author on reasonable request.

\section{Ethical Approval}

Ethical approval was obtained from the ethics committee of the Department of Cardiology, Fuwai Hospital, National Center for Cardiovascular Diseases, Peking Union Medical College, China. 


\section{Conflicts of Interest}

No conflicts of interest are declared by the authors.

\section{Authors' Contributions}

Hongbing Yan, Xiaoxiao Zhao, and WangYing were involved in analyses and model construction and manuscript drafting. All authors were involved in the critical revision of the manuscript for important intellectual content and approval of the final version. Xiaoxiao Zhao and Ying Wang contributed equally to this manuscript.

\section{Acknowledgments}

This study was approved by the Chinese Academy of Medical Sciences Innovation Fund for Medical Sciences (2016-I2M1-009). The authors gratefully acknowledge all individuals who participated in this study.

\section{References}

[1] A. S. Jaffe, J. Ravkilde, R. Roberts et al., "It's time for a change to a troponin standard," Circulation, vol. 102, no. 11, pp. 1216-1220, 2000.

[2] K. Thygesen, J. S. Alpert, A. S. Jaffe, M. L. Simoons, B. R. Chaitman, and H. D. White, "Third universal definition of myocardial infarction," Circulation, vol. 126, no. 16, pp. 2020-2035, 2012.

[3] D. A. Morrow, C. P. Cannon, R. L. Jesse et al., "National academy of clinical biochemistry laboratory medicine practice guidelines: clinical characteristics and utilization of biochemical markers in acute coronary syndromes," Circulation, vol. 115, no. 13, pp. 552-574, 2007.

[4] A. S. V. Shah, A. Anand, F. E. Strachan et al., "High-sensitivity troponin in the evaluation of patients with suspected acute coronary syndrome: a stepped-wedge, cluster-randomised controlled trial," The Lancet, vol. 392, no. 10151, pp. 919-928, 2018.

[5] C. W. L. Chin, A. S. V. Shah, D. A. McAllister et al., "Highsensitivity troponin I concentrations are a marker of an advanced hypertrophic response and adverse outcomes in patients with aortic stenosis," European Heart Journal, vol. 35, no. 34, pp. 2312-2321, 2014.

[6] A. S. V. Shah, D. A. McAllister, R. Mills et al., "Sensitive troponin assay and the classification of myocardial infarction," The American Journal of Medicine, vol. 128, no. 5, pp. 493-501, 2015.

[7] D. N. Feldman, R. M. Minutello, G. Bergman, I. Moussa, and S. C. Wong, "Relation of troponin I levels following nonemergent percutaneous coronary intervention to short- and long-term outcomes," The American Journal of Cardiology, vol. 104, no. 9, pp. 1210-1215, 2009.

[8] P. G. Steg, P. G. Steg, S. K. James et al., "ESC guidelines for the management of acute myocardial infarction in patients presenting with ST-segment elevation," European Heart Journal, vol. 33, no. 20, pp. 2569-2619, 2012.

[9] P. T. O’Gara, F. G. Kushner, D. D. Ascheim et al., “2013 ACCF/AHA guideline for the management of ST-elevation myocardial infarction: executive summary: a report of the American College of Cardiology Foundation/American Heart Association task force on practice guidelines," Catheterization and Cardiovascular Interventions, vol. 82, no. 1, pp. E1-E27, 2013.

[10] B. Ibanez, S. James, S. Agewall et al., "2017 ESC guidelines for the management of acute myocardial infarction in patients presenting with ST-segment elevation: the task force for the management of acute myocardial infarction in patients presenting with ST-segment elevation of the European Society of Cardiology (ESC)," European Heart Journal, vol. 39, pp. 119-177, 2018.

[11] J. A. de Lemos, M. H. Drazner, T. Omland et al., "Association of troponin $\mathrm{T}$ detected with a highly sensitive assay and cardiac structure and mortality risk in the general population," JAMA, vol. 304, no. 22, pp. 2503-2512, 2010.

[12] S. Aeschbacher, T. Schoen, M. Bossard et al., "Relationship between high-sensitivity cardiac troponin I and blood pressure among young and healthy adults," American Journal of Hypertension, vol. 28, no. 6, pp. 789-796, 2014.

[13] L. B. Daniels, G. A. Laughlin, P. Clopton, A. S. Maisel, and E. Barrett-Connor, "Minimally elevated cardiac troponin T and elevated N-terminal pro-B-type natriuretic peptide predict mortality in older adults," Journal of the American College of Cardiology, vol. 52, no. 6, pp. 450-459, 2008.

[14] P. Ml, G. Bonetti, F. Pagani, F. Stefini, R. Giubbini, and C. Cuccia, "Measurement of troponin I $48 \mathrm{~h}$ after admission as a tool to rule out impaired left ventricular function in patients with a first myocardial infarction," Clinical Chemistry and Laboratory Medicine (CCLM), vol. 43, no. 8, pp. 848-854, 2005.

[15] E. Giannitsis, H. Steen, K. Kurz et al., "Cardiac magnetic resonance imaging study for quantification of infarct size comparing directly serial versus single time-point measurements of cardiac troponin T," Journal of the American College of Cardiology, vol. 51, no. 3, pp. 307-314, 2008.

[16] A. C. Rao, P. O. Collinson, A. J. Rose, C. John, R. CanepaAnson, and S. P. Joseph, "Prospective evaluation of the role of routine cardiac troponin $\mathrm{T}$ measurement to identify left ventricular ejection fraction $<40 \%$ after first myocardial infarction," Heart (British Cardiac Society), vol. 89, no. 5, pp. 559-560, 2003.

[17] C. L. Hu, Y. B. Li, Y. G. Zou et al., "Troponin Tmeasurement can predict persistent left ventricular dysfunction in peripartum cardiomyopathy," Heart, vol. 93, no. 4, pp. 488-490, 2007.

[18] J. F. Younger, S. Plein, J. Barth, J. P. Ridgway, S. G. Ball, and J. P. Greenwood, "Troponin-I concentration $72 \mathrm{~h}$ after myocardial infarction correlates with infarct size and presence of microvascular obstruction," Heart (British Cardiac Society), vol. 93, no. 12, pp. 1547-1551, 2007.

[19] M. B. Nienhuis, J. P. Ottervanger, H. J. Bilo, B. D. Dikkeschei, and F. Zijlstra, "Prognostic value of troponin after elective percutaneous coronary intervention: a meta-analysis," Catheterization and Cardiovascular Interventions, vol. 71, pp. 318-324, 2010.

[20] D. N. Feldman, L. Kim, A. G. Rene, R. M. Minutello, G. Bergman, and S. C. Wong, "Prognostic value of cardiac troponin-I or troponin-T elevation following nonemergent percutaneous coronary intervention: a meta-analysis," Catheterization and Cardiovascular Interventions, vol. 77, no. 7, pp. 1020-1030, 2011.

[21] M. K. Christensen, H. Huang, C. Torp-Pedersen, T. Trydal, and J. Ravkilde, "Ravkilde incidence and impact on prognosis of peri-procedural myocardial infarction in 2760 elective patients with stable angina pectoris in a historical prospective follow-up study," Cardiovascular Disorders, vol. 16, p. 140, 2016. 
[22] M. Than, L. Cullen, S. Aldous et al., "2-Hour accelerated diagnostic protocol to assess patients with chest pain symptoms using contemporary troponins as the only biomarker," Journal of the American College of Cardiology, vol. 59, no. 23, pp. 2091-2098, 2012.

[23] S. Matetzky, T. Sharir, M. Domingo et al., "Elevated troponin I level on admission is associated with adverse outcome of primary angioplasty in acute myocardial infarction," Circulation, vol. 102, no. 14, pp. 1611-1616, 2000.

[24] A. De Labriolle, G. Lemesle, L. Bonello et al., "Prognostic significance of small troponin I rise after a successful elective percutaneous coronary intervention of a native artery," The American Journal of Cardiology, vol. 103, no. 5, pp. 639-645, 2009.

[25] M. Sezer, I. Okcular, T. Goren et al., "Association of haematological indices with the degree of microvascular injury in patients with acute anterior wall myocardial infarction treated with primary percutaneous coronary intervention," Heart, vol. 93, no. 3, pp. 313-318, 2007.

[26] N. Kobayashi, A. Maehara, S. J. Brener et al., "Usefulness of the left anterior descending coronary artery wrapping around the left ventricular apex to predict adverse clinical outcomes in patients with anterior wall ST-segment elevation myocardial infarction (from the harmonizing outcomes with revascularization and stents in acute myocardial infarction trial)," The American Journal of Cardiology, vol. 116, no. 11, pp. 1658-1665, 2015.

[27] M. G. McLaughlin, G. W. Stone, E. Aymong et al., "Prognostic utility of comparative methods for assessment of ST-segment resolution after primary angioplasty for acute myocardial infarction," Journal of the American College of Cardiology, vol. 44, no. 6, pp. 1215-1223, 2004.

[28] L. Galiuto, B. Garramone, A. Scarà et al., "The extent of microvascular damage during myocardial contrast echocardiography is superior to other known indexes of post-infarct reperfusion in predicting left ventricular remodeling," Journal of the American College of Cardiology, vol. 51, no. 5, pp. 552-559, 2008.

[29] T. Ashraf, M. N. Khan, S. M. Afaque et al., "Clinical and procedural predictors and short-term survival of the patients with no reflow phenomenon after primary percutaneous coronary intervention," International Journal of Cardiology, vol. 294, pp. 27-31, 2019.

[30] B. K. Nallamothu, E. H. Bradley, and H. M. Krumholz, "Time to treatment in primary percutaneous coronary intervention," New England Journal of Medicine, vol. 357, no. 16, pp. 1631-1638, 2007.

[31] G. Niccoli, F. Burzotta, L. Galiuto, and F. Crea, "Myocardial no-reflow in humans," Journal of the American College of Cardiology, vol. 54, no. 4, pp. 281-292, 2009.

[32] G. W. Stone, B. Witzenbichler, G. Weisz et al., "Platelet reactivity and clinical outcomes after coronary artery implantation of drug-eluting stents (ADAPT-DES): a prospective multicentre registry study," The Lancet, vol. 382, no. 9892, pp. 614-623, 2013.

[33] I. Benedek, M. Gyongyosi, and T. Benedek, "A prospective regional registry of ST-elevation myocardial infarction in central Romania: impact of the stent for life initiative recommendations on patient outcomes," American Heart Journal, vol. 166, no. 3, pp. 457-465, 2013.

[34] E. Abu-Assi, I. Ferreira-González, A. Ribera et al., "Do GRACE (Global Registry of Acute Coronary events) risk scores still maintain their performance for predicting mortality in the era of contemporary management of acute coronary syndromes?" American Heart Journal, vol. 160, no. 5 , pp. $826-834,2010$.

[35] B. Elbarouni, S. G. Goodman, R. T. Yan et al., "Validation of the global registry of acute coronary event (GRACE) risk score for in-hospital mortality in patients with acute coronary syndrome in Canada," American Heart Journal, vol. 158, no. 3, pp. 392-399, 2009.

[36] P. Anna, S. De Rosa, M. R. García et al., "Experimental modeling and identification of cardiac biomarkers release in acute myocardial infarction," Transactions on Control Systems Technology, vol. 28, no. 1, pp. 1-13, 2018. 